\title{
Protective effects of p-nitro caffeic acid phenethyl ester on acute myocardial ischemia-reperfusion injury in rats
}

\author{
QIN DU, CHUNZHI HAO, JING GOU, XIAOLI LI, KAILI ZOU, XIAOYAN HE and ZHUBO LI \\ College of Pharmaceutical Sciences and Chinese Medicine, Southwest University, Chongqing 400716, P.R. China
}

Received February 11, 2015; Accepted October 13, 2015

DOI: $10.3892 /$ etm. 2016.3070

\begin{abstract}
Myocardial ischemia-reperfusion (IR) causes widespread cardiomyocyte dysfunction, including apoptosis and necrosis. The present study aimed to investigate the possible cardioprotective effects of p-nitro caffeic acid phenethyl ester $\left(\mathrm{CAPE}-\mathrm{NO}_{2}\right)$ on myocardial IR-induced injury in vivo. To generate a rat model of myocardial IR, the left anterior descending coronary artery was occluded for $30 \mathrm{~min}$, followed by reperfusion for $2 \mathrm{~h}$. The rats were administered either the sham treatment (the sham and IR control groups) or the therapeutic agents [the caffeic acid phenethyl ester (CAPE) and CAPE- $\mathrm{NO}_{2}$ groups] 10 min prior to the occlusion. Myocardial IR-induced injury is characterized by: A significant increase in the levels of myocardial enzymes, including creatine kinase, lactate dehydrogenase and aspartate transaminase; a marked increase in intercellular adhesion molecule 1 expression levels, lipid peroxidation products and inflammatory mediators; and a significant decrease in myocardial antioxidants, including catalase, total superoxide dismutase and glutathione peroxidase. In the present study, pretreatment with CAPE-NO significantly ameliorated these changes, and decreased the infarct size, as compared with the IR control group $(10.32 \pm 3.8$ vs. $35.65 \pm 5.4 \%)$. Furthermore, western blotting demonstrated that pretreatment with $\mathrm{CAPE}-\mathrm{NO}_{2}$ downregulated the myocardial IR-induced protein expression levels of B-cell lymphoma-2 (Bcl-2)-associated X protein (Bax), cleaved caspase-3, P38 and the Bax/Bcl-2 ratio. CAPE-NO also upregulated the myocardial IR-induced expression levels of Bcl-2, phosphoinositide-3-kinase, phosphorylated Akt and mammalian target of rapamycin. In conclusion, the results of the present study indicated that CAPE-NO $\mathrm{N}_{2}$ demonstrated improved cardioprotective effects, as compared with CAPE;
\end{abstract}

Correspondence to: Professor Zhubo Li, College of Pharmaceutical Sciences and Chinese Medicine, Southwest University, 2 Tiansheng Road, Chongqing 400716, P.R. China

E-mail: lizhubo2007@163.com

Key words: caffeic acid phenethyl ester, derivative, oxidative stress, inflammation, phosphoinositide-3-kinase/Akt/mammalian target of rapamycin signaling pathway therefore, $\mathrm{CAPE}-\mathrm{NO}_{2}$ may represent a novel approach to pharmacological cardioprotection.

\section{Introduction}

Acute myocardial infarction is a leading cause of human mortality worldwide (1-2). In the clinic, restoration of blood flow is the most effective way to prevent necrosis of the myocardium. However, myocardial ischemia-reperfusion (IR) injuries including arrhythmias, myocardial stunning, and microvascular damage may also occur when blood flow is restored (1-4).

Two major factors, oxidative stress and inflammatory response, have vital roles in the mechanisms of necrosis and apoptosis, and are also involved in the pathogenesis of IR injury (1-3). Oxidative stress is usually associated with the formation of reactive oxygen species (ROS), which may trigger a cytotoxic cascade leading to the activation of various downstream targets and subsequent inflammation and lipid peroxidation $(2,3)$. Previous studies have demonstrated that activation of the phosphoinositide-3-kinase (PI3K)/Akt pathway may protect against IR-induced myocardial injury and prevent apoptosis $(2,4,5)$. In addition to regulating cell survival through the phosphorylation of various anti-apoptotic proteins, such as Akt and mammalian target of rapamycin (mTOR), PI3K can also regulate and control several downstream targets, including B-cell lymphoma (Bcl)-2 and caspases. Furthermore, the pro-apoptotic protein, P38, which is a member of the mitogen-activated protein kinase pathway, has a crucial role in cell growth and survival (6). Strengthening the antioxidant defenses and inhibiting the apoptotic pathway may be important for reducing myocardial IR damage.

Caffeic acid phenethyl ester (CAPE; Fig. 1A) is a small, flavonoid-like compound isolated from honey bee propolis, which possesses high anti-inflammatory, antioxidant, free radical scavenging and anti-platelet aggregation activity, and has protective effects against IR injury $(1,7,8)$. The authors of the present study have previously designed and synthesized a series of CAPE derivatives (8). Among these derivatives, p-nitro caffeic acid phenethyl ester (CAPE-NO ${ }_{2}$; Fig. 1B) possessed the highest activity levels against platelet aggregation and the highest capability to increase the white blood cell count, nitric oxide production, and spleen and thymic indices.

Several therapeutic agents used in the treatment of cardiac disease, such as nifedipine and imodipine, contain unsaturated 
p-nitro and phenyl moieties. In light of the structural similarities demonstrated between these therapeutic agents and CAPE- $\mathrm{NO}_{2}$, the authors of the present study hypothesized that this novel derivative may provide increased protection against IR-induced myocardial injury in vivo. In the present study, a rat model of myocardial IR-induced injury was generated in order to investigate the protective effects and mechanisms of CAPE- $\mathrm{NO}_{2}$.

\section{Materials and methods}

Materials. CAPE and CAPE- $\mathrm{NO}_{2}$ (purity, >99.0\%) were synthesized as previously described (8). 2,3,5-triphenyltetrazolium chloride (TTC) was purchased from Amresco, LLC (Solon, OH, USA). Diagnostic assay kits for analyzing creatine kinase $(\mathrm{CK})$, lactate dehydrogenase (LDH), aspartate transaminase (AST), total superoxide dismutase (T-SOD), catalase (CAT), glutathione-peroxidase (GSH-Px), malondialdehyde (MDA) and myeloperoxidase (MPO) were purchased from Nanjing Jiancheng Bioengineering Institute (Nanjing, China). Anti-intercellular adhesion molecule (ICAM)-1 (GTX48197-PRO) antibody was purchased from Dako (Glostrup, Denmark). Antibodies against PI3K (21890-1-AP), phosphorylated PI3K (p-PI3K; 17894-1-AP), Akt (10176-2-AP), p-Akt (60072-1-1G), mTOR (10176-2-AP), Bcl-2 (12789-1-AP), Bax (50599-2-1G), cleaved caspase-3 (25546-1-AP) and P38 (14064-1-AP), and horseradish peroxidase (HRP)-conjugated goat anti-rabbit secondary antibody (SA-00001) and Tris-buffered saline were purchased from Proteintech Group, Inc (Chicago, IL, USA). Anti-p-mTOR (BS-4706) was purchased from Bioworld Technology, Inc. (St. Louis Park, MN, USA). Anti- $\beta$-actin antibody (AC001-R) and Tween-20 were purchased from Beijing Dingguo Changsheng Biotechnology Co., Ltd (Beijing, China). All other chemicals used in the present study were of analytical grade.

Experimental animals and treatment. The present study was approved by the Ethics Committee for Animal Experimentation of Chongqing Medical University (Chongqing, China).

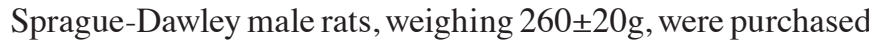
from the Experimental Animal Center of Chongqing Medical University (SCXK(YU)2012-0001). The rats were maintained under standard conditions of humidity $(50 \%)$ and temperature $\left(25 \pm 2^{\circ} \mathrm{C}\right)$ in a $12 \mathrm{~h}$ light and dark cycle. The rats were fed standard rodent chow, with ad libitum access to water, and were acclimated for at least one week prior to experimentation.

The rats were randomly assigned to four groups, with 12 rats in each group: (i) The sham group, rats were intravenously injected (i.v.) with the vehicle (dimethyl sulfoxide diluted in $0.9 \% \mathrm{NaCl}, \mathrm{v} / \mathrm{v}=1: 104) 10 \mathrm{~min}$ prior to and during the sham occlusion surgery at a dose of $1 \mathrm{ml} / \mathrm{kg}$ body weight; (ii) the IR control group, rats were administered with the vehicle ( $1 \mathrm{ml} / \mathrm{kg}$ body weight,i.v.) $10 \mathrm{~min}$ prior to and during the occlusion; (iii) the CAPE group, rats were administered with CAPE (10 $\mu \mathrm{g} / \mathrm{kg}$ body weight, i.v.) $10 \mathrm{~min}$ prior to and during the occlusion; and (iv) the CAPE- $\mathrm{NO}_{2}$ group, rats were administered with CAPE- $\mathrm{NO}_{2}(10 \mu \mathrm{g} / \mathrm{kg}$ body weight, i.v. $) 10 \mathrm{~min}$ prior to and during the occlusion. CAPE and CAPE-NO $\mathrm{N}_{2}$ were dissolved in the vehicle to a final concentration of $10 \mu \mathrm{g} / \mathrm{ml}$.
Myocardial IR procedure. The rats were anesthetized with $40 \mathrm{mg} / \mathrm{kg}$ sodium pentobarbital, administered intraperitoneally. Following a tracheotomy, ventilation was provided using a breathing machine (respiratory rate, 60 breaths/min; tidal volume, $10-12 \mathrm{ml} / \mathrm{kg}$ ) and heart rate was monitored during the procedure using an electrocardiogram (ECG). The myocardial IR operation was conducted as described previously (2). Briefly, the left anterior descending (LAD) coronary artery was ligated using a 6-0 silk suture. Following this, a medical latex tube (inner diameter, $1.5 \mathrm{~mm}$ ) was placed between the ligature and the LAD. Myocardial ischemia was induced by tightening the ligature around the latex tube to compress the LAD. Significant ECG changes, including elevation of the ST segment and widening of the QRS complex, indicated that the coronary occlusion was successful. Following $30 \mathrm{~min}$ of ischemia, the latex tube was removed in order to restore the coronary circulation. The sham group underwent the same procedures, with the exception that the silk suture was left untied.

Following $2 \mathrm{~h}$ reperfusion, all of the rats were sacrificed using $10 \%$ chloral hydrate (0.3-0.4 ml/100 g body weight) and blood samples were subsequently collected in order to obtain the serum for further biochemical analysis. The heart tissues were quickly removed, washed with ice-cold normal saline, blotted dry on filter paper and weighed. Several sections of the tissues were used for measuring the infarct size and preparing homogenates; whereas the remaining tissues were used for immunohistochemical and histopathological analyses.

Quantification of infarct size. Myocardial infarct size was measured using the TTC staining method as previously described (3). The rat hearts were maintained at $-20^{\circ} \mathrm{C}$. The ventricles were sliced into $5 \mathrm{~mm}$ cross sectional rings along the short axis. The sections were weighed and incubated in $2 \%$ TTC (pH 7.4) for $15 \mathrm{~min}$ at $37^{\circ} \mathrm{C}$ in the dark. The normal tissues were subsequently stained red, whereas the infarcted tissues remained unstained (white or pale). The infarcted area was demarcated, and its size was analyzed using Image-Pro Plus 7.0 software (Media Cybernetics, Inc., Rockville, MD, USA). As a percentage of left ventricular mass, infarct size was calculated as the ratio of infarcted myocardium to the risk region $\mathrm{x} 100 \%$.

Examination of histopathology. Heart tissue sections (5 mm) were fixed in $10 \%$ buffered formalin for $24 \mathrm{~h}$ and subsequently embedded in paraffin. Following staining with hematoxylin and eosin (Wuhan Goodbio Technology Co., Ltd., Wuhan, China), the sections were examined using a Nikon TE2000 light microscope (Nikon Corporation, Tokyo, Japan).

Preparation of cardiac homogenate. Half of the heart tissues were rinsed in ice-cold normal saline and homogenized in Tris- $\mathrm{HCl}$ buffer $(0.01 \mathrm{M}, \mathrm{pH} 7.4)$ at $10,000 \mathrm{rpm}$ at $4^{\circ} \mathrm{C}$ for $5 \mathrm{~min}$ using an electric homogenizer (Ningbo Scientz Biotechnology Co., Ltd., Ningbo, China) to obtain $10 \%$ homogenates. The homogenates were subsequently centrifuged at $10,000 \mathrm{xg}$ at $4^{\circ} \mathrm{C}$ for $10 \mathrm{~min}$, and the resultant supernatants were collected in order to measure the levels of CAT, T-SOD, GSH-Px, MPO and MDA. 
<smiles>O=C(/C=C/c1ccc(O)c(O)c1)OCCc1ccccc1</smiles>

B<smiles>O=C(/C=C/c1ccc(O)c(O)c1)OCCc1ccc([N+](=O)[O-])cc1</smiles>

Figure 1. Chemical structures of (A) p-nitro caffeic acid phenethyl ester and (B) caffeic acid phenethyl ester.

Measurement of cardiac marker enzymes in the serum. Serum samples were isolated from the blood samples by centrifugation at $650 \mathrm{x}$ g for $15 \mathrm{~min}$. Serum AST, LDH and $\mathrm{CK}$ levels were measured using spectrophotometric kits, according to the manufacturer's protocol.

Measurement of the antioxidant system and lipid peroxidation products. The heart homogenates were analyzed for lipid peroxidation, by proxy of the MDA content, and the levels of endogenous anti-peroxidative enzymes, including CAT, T-SOD and GSH-Px, were measured using spectrophotometric kits according to the respective manufacturer's protocols.

Measurement of inflammatory mediators. MPO levels were measured using a spectrophotometric kit. ICAM-1 expression levels were determined via immunohistochemical staining, according to the manufacturer's protocol.

Immunohistochemical staining. The formalin-fixed, paraffin-embedded heart tissue sections $(5 \mathrm{~mm})$ were de-paraffinized and subsequently rehydrated. Endogenous peroxidase activity was blocked using $3 \% \mathrm{H}_{2} \mathrm{O}_{2}$, and following microwave antigen retrieval using $1 \mathrm{mM}$ EDTA-Tris bugger ( $\mathrm{pH}$ 9.0; Wuhan Goodbio Technology Co., Ltd.), the sections were incubated with $10 \%$ normal goat serum (Wuhan Boster Biotechnology Co., Ltd., Wuhan, China), and then overnight at $4^{\circ} \mathrm{C}$ with rabbit anti-ICAM-1 antibody. The samples were subsequently washed and HRP-conjugated goat anti-rabbit IgG (1:5,000; SH-0032; Beijing Dingguo Changsheng Biotechnology Co., Ltd.) secondary antibodies were applied at $37^{\circ} \mathrm{C}$ for $50 \mathrm{~min}$. Finally, diaminobenzidine was used for color development, and counterstaining was performed using hematoxylin. Images were obtained using a light microscope (magnification, 100x; Nikon Corporation) and were subsequently assessed by densitometry using Image-Pro Plus 6.0 software (Media Cybernetics, Inc.). The calculated densities correlate with the expression levels of the protein.
Western blot analysis. Myocardium tissues were homogenized using RIPA lysis buffer (Nanjing Jiancheng Institute of Biotechnology) at $4^{\circ} \mathrm{C}$ for $30 \mathrm{~min}$, centrifuged at $12,000 \mathrm{x} \mathrm{g}$ for $10 \mathrm{~min}$ and the supernatant was subsequently removed. Proteins were quantified using the BCA kit (Beyotime Institute of Biotechnology, Haimen, China) and were subsequently separated by $12 \%$ sodium dodecyl sulfate polyacrylamide gel electrophoresis and transferred to polyvinylidene difluoride membranes (Nanjing Jiancheng Institute of Biotechnology). Following this, the membranes were blocked with 5\% non-fat dried milk and incubated with primary antibodies against Akt :1,000), p-Akt (1:1,000), PI3K (1:500), p-PI3K (1:500), mTOR (1:500), p-mTOR (1:500), Bcl-2 (1:1,000), Bax (1:500), cleaved caspase-3 (1:500) and P38 (1:500) and $\beta$-actin $(1: 2,000)$ overnight at $4^{\circ} \mathrm{C}$. Subsequently, the membranes were washed three times with Tris-buffered saline with Tween-20 for $10 \mathrm{~min}$, incubated with HRP-conjugated goat anti-rabbit secondary antibody $(1: 5,000)$ for $2 \mathrm{~h}$ at room temperature, and washed again. The protein bands were developed using a chemiluminescent system and subsequently quantified via densitometric analysis using Quantity One (both Bio-Rad Laboratories Inc., Hercules, CA, USA).

Statistical analysis. Data are expressed as the mean \pm standard deviation. Statistical significance was determined using one-way analysis of variance followed by least significant difference multiple comparison tests using SPSS 18.0 software (SPSS, Inc., Chicago, IL, USA). P $<0.05$ was considered to indicate a statistically significant difference.

\section{Results}

Reduction of the myocardial infarct size. As outlined in Fig. 2A and B, minimal myocardial tissues with a risk of infarction were detected in the sham group (infarct size, $1.1 \pm 0.4 \%$ ); whereas IR resulted in myocardial infarction with a mean infarct size of $35.65 \pm 5.4 \%$. Treatment with CAPE and CAPE- $\mathrm{NO}_{2}$ markedly decreased the infarct size to $14.7 \pm 3.14 \%$ and $10.32 \pm 3.8 \%$, respectively. These results indicate that CAPE- $\mathrm{NO}_{2}$ may significantly increase $(\mathrm{P}<0.05)$ protection against myocardial IR injury, as compared with CAPE.

Myocardial necrosis following IR. The effects of CAPE and CAPE- $\mathrm{NO}_{2}$ administration on myocardial histopathological damage were observed. In the sham group (Fig. 3A), the cardiac muscle fibers were relatively uniform, with little inflammatory infiltration, edema or cardiac necrosis. In the IR control group (Fig. 3B), ischemia resulted in histological changes in cardiac morphology, including massive cell necrosis and the loss of cardiomyocyte architecture on the left ventricular wall. Treatment with CAPE-NO (Fig. 3C) and CAPE (Fig. 3D) markedly alleviated this histopathological damage.

Determination of cardiac marker enzyme activity levels. As outlined in Fig. 4A-C, the activities of CK, LDH and AST were significantly increased in the IR control group, as compared with the sham group. Pretreatment with CAPE and CAPE- $\mathrm{NO}_{2}$ significantly inhibited the elevation of $\mathrm{CK}$ (by 48.6 and $59.3 \%$, respectively), LDH (by 30.2 and $55.1 \%$, 

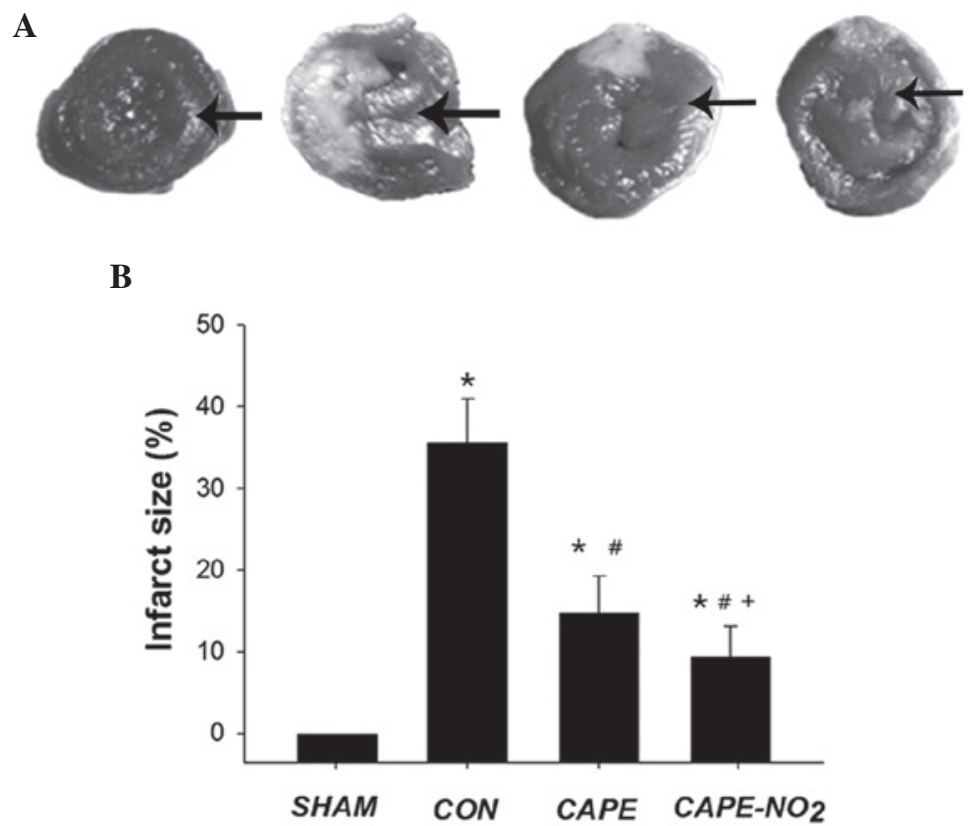

Figure 2. Effects of CAPE and CAPE- $\mathrm{NO}_{2}$ in reducing infarct size in the heart of a rat model with induced acute ischemia-reperfusion injury. (A) Representative images of TTC-stained slices of the left ventricle of the rat heart. The deep red-stained areas (arrows) indicate normal tissue, whereas the unstained pale areas indicate infarcted tissue. (B) Myocardial infarct size determined by TTC staining. Data are expressed as the mean \pm standard deviation ( $\mathrm{n}=12)$. ${ }^{*} \mathrm{P}<0.05$ vs. the sham group; ${ }^{\#} \mathrm{P}<0.05$ vs. the CON group; and ${ }^{+} \mathrm{P}<0.05$ vs. the CAPE group. TTC, 2,3,5-triphenyltetrazolium chloride; CON, ischemia-reperfusion control group; CAPE, caffeic acid phenethyl ester; CAPE- $\mathrm{NO}_{2}$, p-nitro caffeic acid phenethyl ester.

A

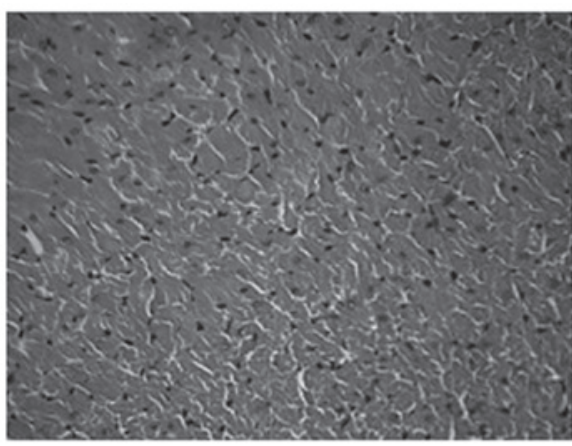

C

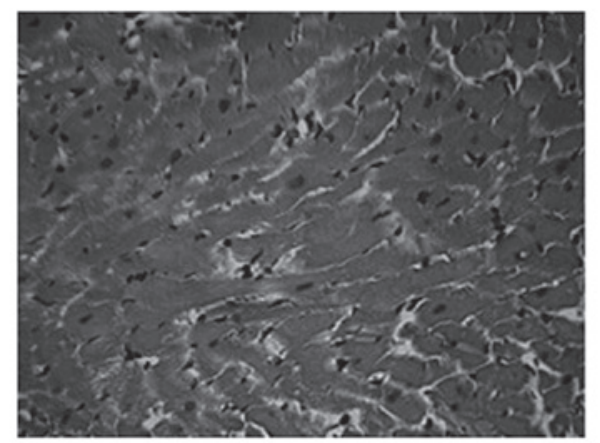

B

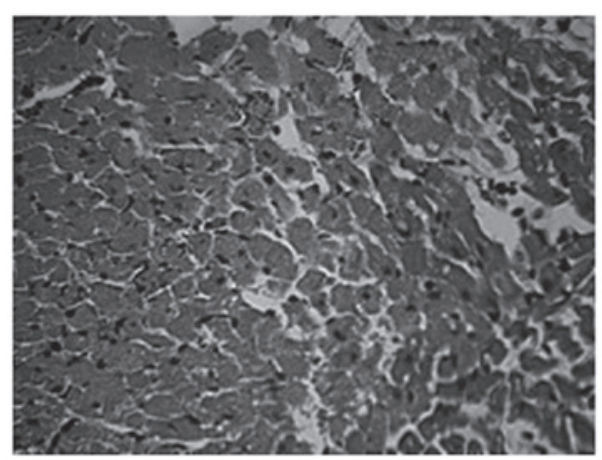

D

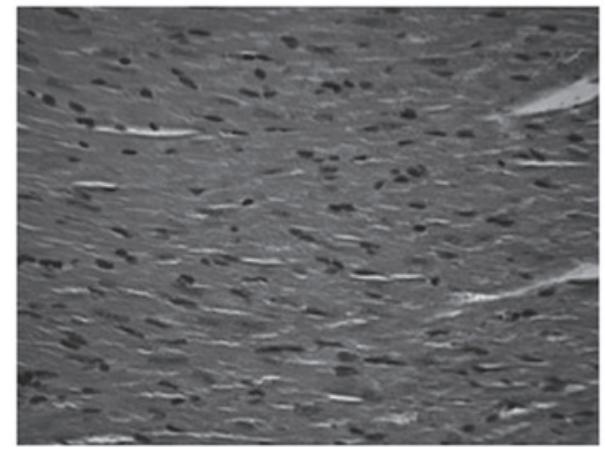

Figure 3. Effects of CAPE and CAPE- $\mathrm{NO}_{2}$ administration on myocardial histopathology, assessed by staining with hematoxylin and eosin (magnification, 400x). (A) Sham, (B) ischemia-reperfusion control, (C) CAPE, and (D) CAPE- $\mathrm{NO}_{2}$ groups. CAPE, caffeic acid phenethyl ester; CAPE-NO 2 , p-nitro caffeic acid phenethyl ester.

respectively) and AST activity levels (by 31.2 and $48.1 \%$, respectively) $(\mathrm{P}<0.05$ for all differences). Furthermore, CAPE- $-\mathrm{NO}_{2}$ exhibited a significantly superior protective effect in reducing the activities of CK, LDH and AST $(\mathrm{P}<0.05)$, as compared with CAPE.
Effects of CAPE-NO $\mathrm{O}_{2}$ on the antioxidant system and lipid peroxidation. Ischemia resulted in a significant decrease in CAT, T-SOD and GSH-Px activities, and an increase in the levels of MDA, as compared with the sham group (Fig. 5A-D; $\mathrm{P}<0.05)$. Conversely, pretreatment with CAPE and CAPE- $\mathrm{NO}_{2}$, 

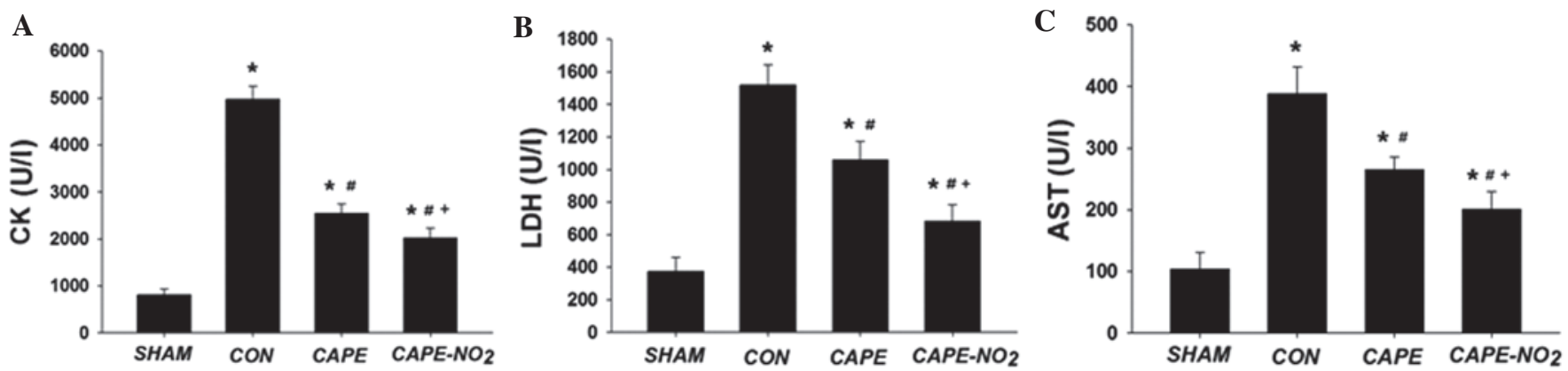

Figure 4. Effects of CAPE and CAPE- $\mathrm{NO}_{2}$ administration on cardiac (A) CK, (B) LDH and (C) AST levels in various groups. Data are expressed as the mean \pm standard deviation $(n=12) .{ }^{*} \mathrm{P}<0.05$ vs. the sham group; ${ }^{*} \mathrm{P}<0.05$ vs. the ischemia/reperfusion control group; ${ }^{+} \mathrm{P}<0.05$ vs. the CAPE group. Con, ischemia/reperfusion control group; CAPE, caffeic acid phenethyl ester; $\mathrm{CAPE}-\mathrm{NO}_{2}$, p-nitro caffeic acid phenethyl ester.
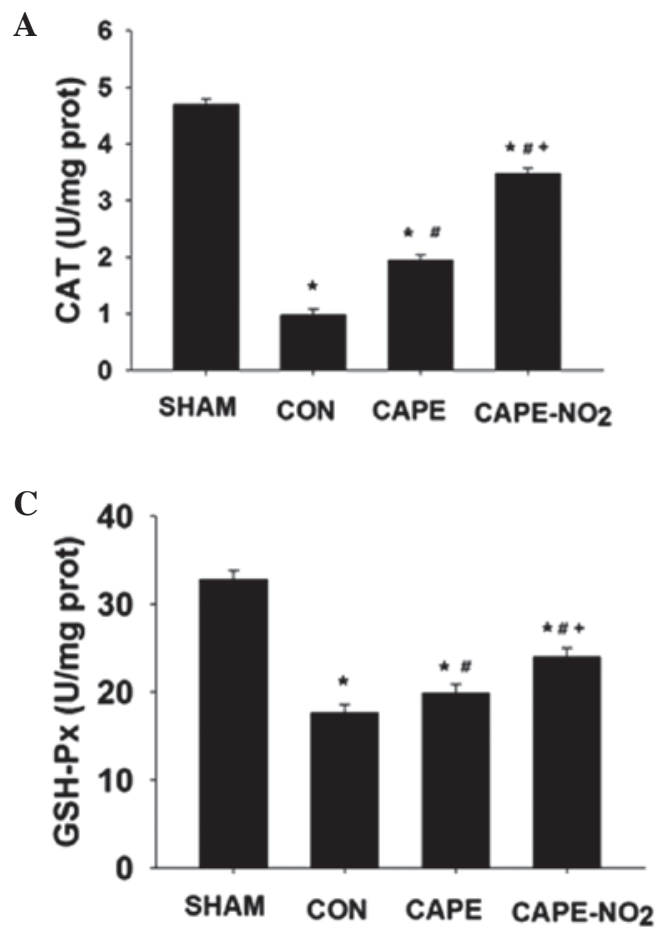

B

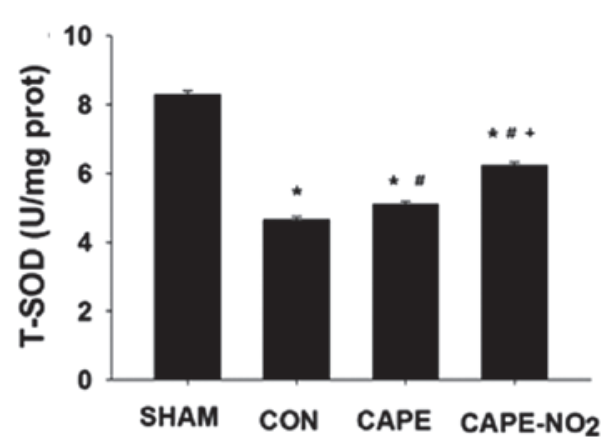

D

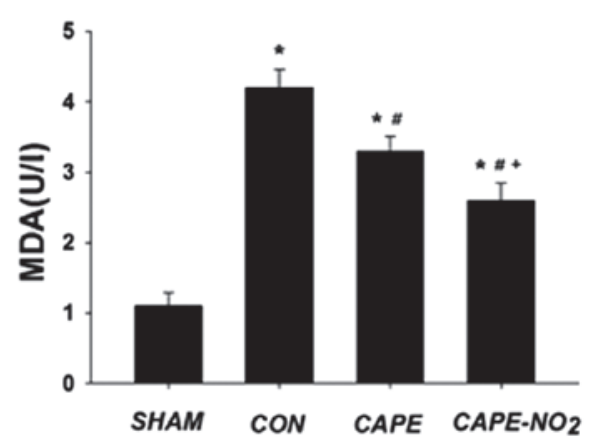

Figure 5. CAPE and CAPE- $\mathrm{NO}_{2}$ enhanced the levels of antioxidant enzymes, including (A) CAT, (B) T-SOD, (C) GSH-Px levels and reduced (D) MDA levels in the various groups. Data are presented as the mean \pm standard deviation $(\mathrm{n}=12) .{ }^{*} \mathrm{P}<0.05$ vs. the sham group; ${ }^{*} \mathrm{P}<0.05$ vs. the CON group; ${ }^{+} \mathrm{P}<0.05$ vs. the $\mathrm{CAPE}$ group. CAT, catalase; T-SOD, total superoxide dismutase; GSH-Px, glutathione-peroxidase; MDA, malondialdehyde; CON, ischemia-reperfusion control group; CAPE, caffeic acid phenethyl ester; $\mathrm{CAPE}-\mathrm{NO}_{2}$, p-nitro caffeic acid phenethyl ester.

significantly ameliorated these alterations $(\mathrm{P}<0.05)$. Furthermore, treatment with CAPE-NO $\mathrm{N}_{2}$ demonstrated greater antioxidant effects, as compared with CAPE.

Anti-inflammatory effects. The expression levels of MPO and ICAM-1 were significantly upregulated in the IR control group, whereas downregulation was demonstrated in the CAPE and CAPE-NO $\mathrm{NO}_{2}$ groups (MPO by 43.9 and $57.1 \%$, respectively, and ICAM-1 by 23.56 and $43.4 \%$, respectively; $\mathrm{P}<0.05$ ) (Fig. 6A and B). These results suggest that $\mathrm{CAPE}-\mathrm{NO}_{2}$ may possess stronger anti-inflammatory activity as compared with CAPE.

Protein expression levels in cardiac tissue. Increased expression levels of Bax, cleaved-caspase- 3 and P38 protein and decreased expression levels of Bcl-2 were demonstrated in the IR control group, as compared with the sham group (Fig. 7A-C; $\mathrm{P}<0.01$ ). Furthermore, upon CAPE and CAPE- $\mathrm{NO}_{2}$ pretreatment, decreased expression levels of Bax, cleaved-caspase-3 and P38 ( $<<0.01)$ and an increase in the expression levels of $\mathrm{Bcl}-2(\mathrm{P}<0.01)$ were demonstrated, as compared with the IR group. In addition, calculation of the relative ratios of $\mathrm{Bax}$ to $\mathrm{Bcl}-2$ (Fig. 7A) demonstrated that $\mathrm{Bax} / \mathrm{Bcl}-2$ in the IR group was significantly increased, as compared with the sham group. The $\mathrm{Bax} / \mathrm{Bcl}-2$ ratios in the CAPE and CAPE- $\mathrm{NO}_{2}$ groups were markedly lower than those in the IR group.

In the IR control group, the protein expression levels of p-PI3K, p-Akt and p-mTOR were significantly decreased $(\mathrm{P}<0.01)$, as compared with the sham group (Fig. 8A-C). Furthermore, the administration of CAPE and CAPE-NO ${ }_{2}$ significantly increased p-PI3K, p-Akt 

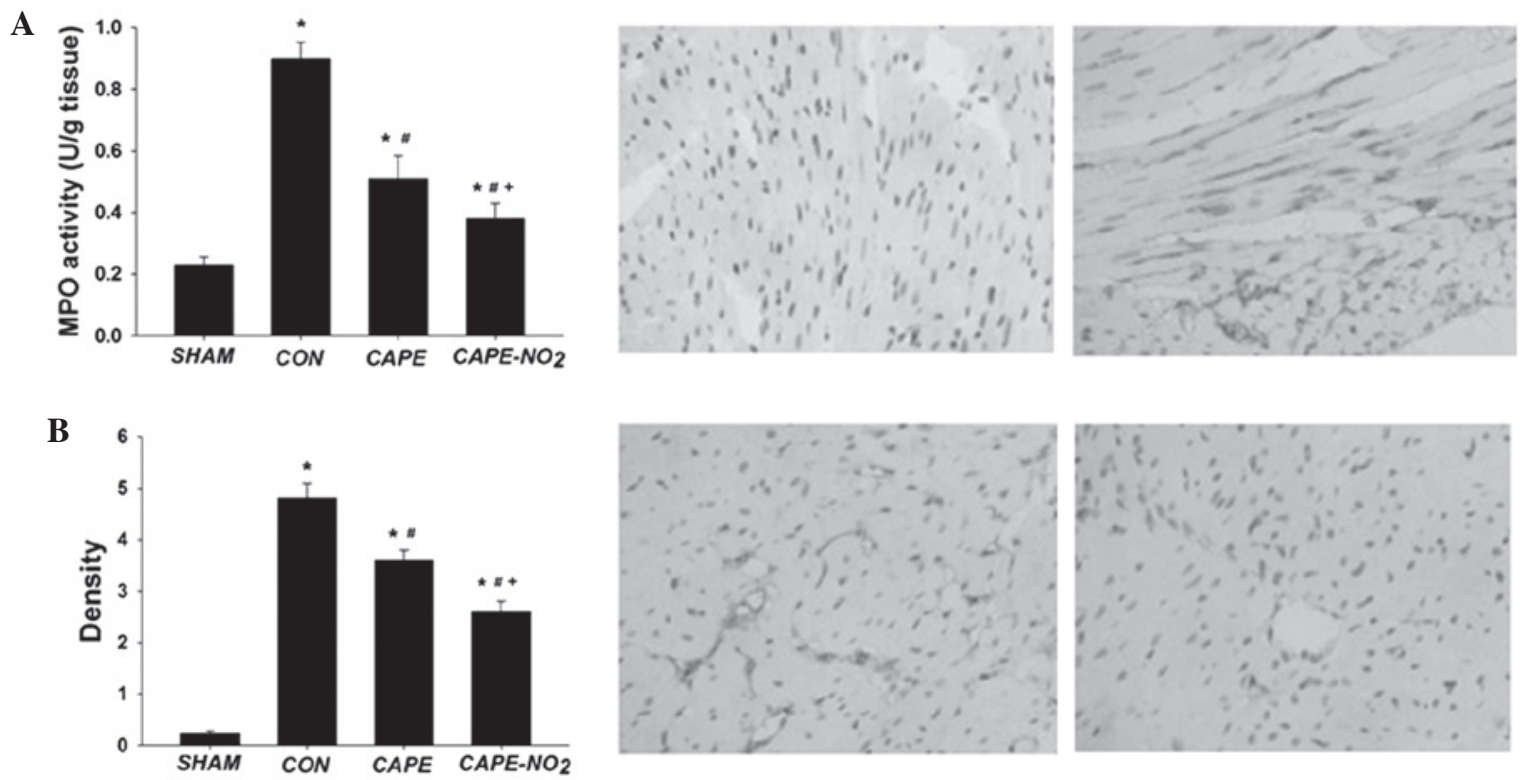

Figure 6. Effects of CAPE and CAPE- $\mathrm{NO}_{2}$ administration on myocardial (A) MPO activity and (B) ICAM-1 expression levels in the various groups. Data are presented as the mean \pm standard deviation $(\mathrm{n}=12)$. ${ }^{*} \mathrm{P}<0.05$ vs. the sham group; ${ }^{*} \mathrm{P}<0.05$ vs. the $\mathrm{CON}$ group; ${ }^{+} \mathrm{P}<0.05$ vs. the CAPE group. CON, ischemia-reperfusion control group; CAPE, caffeic acid phenethyl ester; $\mathrm{CAPE}-\mathrm{NO}_{2}$, p-nitro caffeic acid phenethyl ester.

A

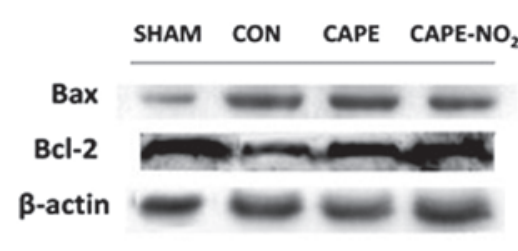

B

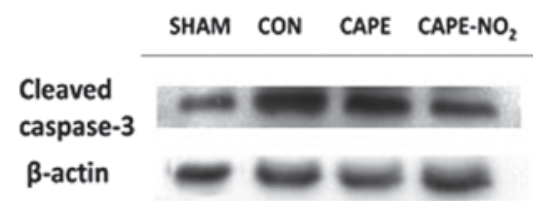

C

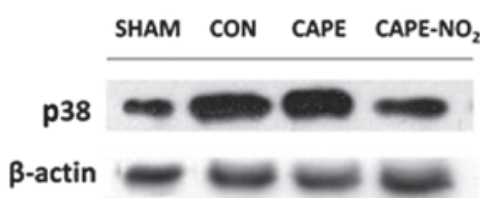

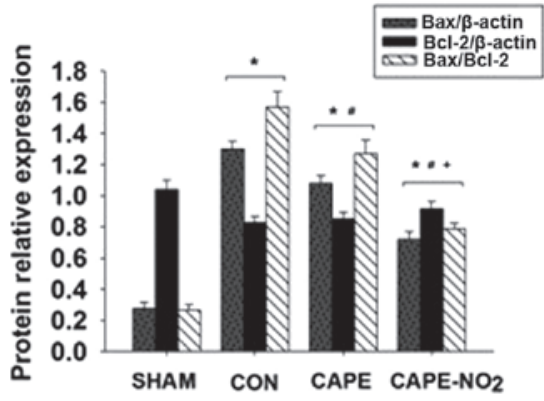
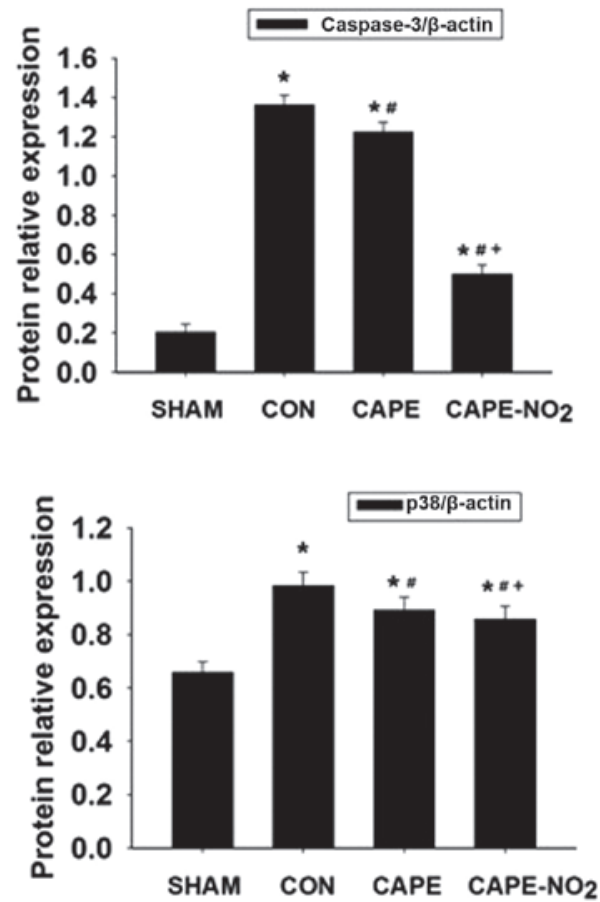

Figure 7. Effects of CAPE and CAPE- $\mathrm{NO}_{2}$ administration on myocardial protein expression levels of (A) Bax and Bcl-2, (B) cleaved-caspase-3 and (C) P38, as well as (A) the Bax/Bcl-2 ratio in the various groups. $\beta$-actin was used as an internal control. Data are expressed as the mean \pm standard deviation (n=12). ${ }^{*} \mathrm{P}<0.05$ vs. the sham group; ${ }^{*} \mathrm{P}<0.05$ vs. the $\mathrm{CON}$ group; ${ }^{+} \mathrm{P}<0.05$ vs. the CAPE group. $\mathrm{CON}$, ischemia-reperfusion control group; CAPE, caffeic acid phenethyl ester; CAPE- $\mathrm{NO}_{2}$, p-nitro caffeic acid phenethyl ester. 
A

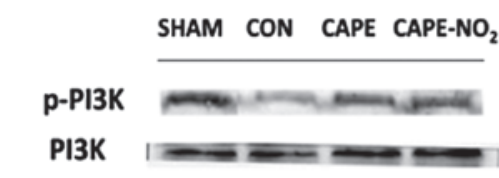

B

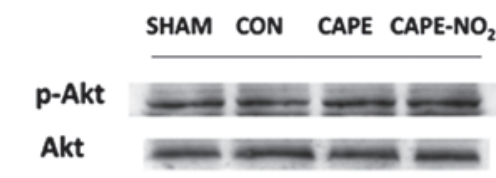

C

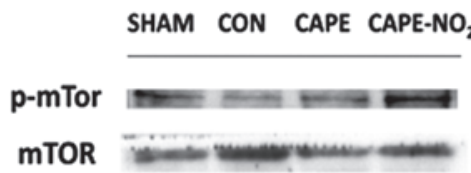

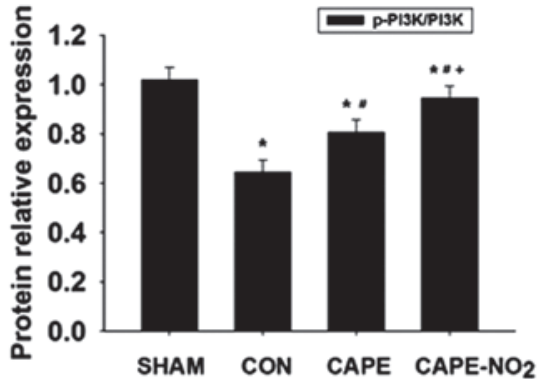
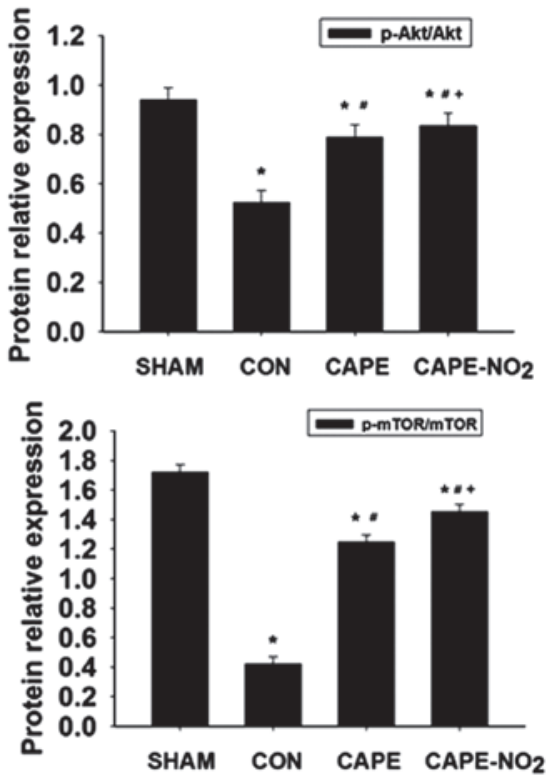

Figure 8. Effects of CAPE and CAPE- $\mathrm{NO}_{2}$ administration on myocardial protein expression levels of (A) PI3K and p-PI3K, (B) p-Akt and Akt, (C) mTORand $\mathrm{p}$-mTOR in the various groups. Data are expressed as the mean \pm standard deviation $(\mathrm{n}=12) .{ }^{*} \mathrm{P}<0.05$ vs. the sham group; ${ }^{\#} \mathrm{P}<0.05$ vs. the $\mathrm{CON}$ group; ${ }^{+} \mathrm{P}<0.05$ vs. the CAPE group. CON, ischemia-reperfusion control group; CAPE, caffeic acid phenethyl ester; $\mathrm{CAPE}-\mathrm{NO}_{2}$, p-nitro caffeic acid phenethyl ester; p, phosphorylated; PI3K, phosphoinositide-3-kinase; mTOR, mammalian target of rapamycin.

and p-mTOR expression levels, as compared with the IR group $(\mathrm{P}<0.01)$.

\section{Discussion}

The present study demonstrated that CAPE- $\mathrm{NO}_{2}$ exerted a protective effect on a rat model of acute myocardial IR injury as indicated by: Significant histopathological alterations, infarct size reductions, restoration of antioxidant enzyme activity levels, amelioration of inflammation and inhibition of apoptosis, as compared with the IR group.

CK, LDH and AST are three myocardial enzymes that are widely used for ascertaining the degree of myocardial injury, and their respective activities were significantly increased in the myocardium of the rats following IR. Pretreatment with CAPE- $\mathrm{NO}_{2}$ significantly inhibited the elevation of $\mathrm{CK}, \mathrm{LDH}$ and AST levels, which suggests that CAPE-NO $\mathrm{N}_{2}$ may be useful as a protective treatment for myocardial IR.

Myocardial infarct size is the gold standard for evaluating the cardioprotective capability of a therapeutic agent (9). As hypothesized, CAPE- $\mathrm{NO}_{2}$ was able to abolish the increase in infarct size demonstrated in the IR group. Furthermore, the results of the present study indicated that CAPE- $\mathrm{NO}_{2}$ further increased myocardial survival and protected cardiac function following myocardial ischemia, as compared with the CAPE group.
Myocardial IR induces severe cardiac damage via necrotic and apoptotic myocardial injury. In addition, oxidative stress resulting from the metabolism of ROS has an integral role in myocardial injury (10). Free radicals are capable of altering the structural and functional integrity of cells through various mechanisms, including lipid peroxidation, proteolysis, and the shearing of nuclear material. A dynamic relationship exists between ROS and antioxidants in the human body (11); however, during myocardial IR, this balance is challenged by the increased demand placed upon the antioxidant defense system (2). Practical therapeutic strategies that may prevent IR-induced myocardial injury include inhibiting the propagation of the oxidative chain reaction and directly decreasing the levels of free radicals (1-3). Furthermore, flavonoid antioxidants are able to protect against oxidative stress and may potentially be used to treat cardiovascular disease (3). As a flavonoid-like compound, CAPE- $\mathrm{NO}_{2}$ has yet to be studied with regards to its antioxidant activity. The present study demonstrated that IR-induced myocardial injury is associated with increased oxidative stress, as indicated by the depletion of endogenous myocardial antioxidants, including CAT, SOD and GSH-Px. Conversely, IR injury-induced ROS were directly scavenged by $\mathrm{CAPE}-\mathrm{NO}_{2}$, thereby preventing lipid peroxidation via MDA and helping to maintain membrane integrity. This effect was further supported by the decrease in CK, LDH and 
AST expression levels demonstrated in the CAPE-NO $\mathrm{N}_{2}$ group. Similarly, CAPE- $\mathrm{NO}_{2}$ effectively blocked the increase in MDA levels, and increased the cardiac activity levels of CAT, SOD and GSH-Px.

Inflammation is a vital pathological mechanism which underlies the propagation of IR-induced myocardial injury (12). MPO and ICAM-1 are inflammatory mediators and markers which are associated with the development of certain inflammatory diseases. Antioxidants attenuate inflammatory responses, suggesting a link between oxidative stress and inflammation (13). In the present study, pretreatment with CAPE- $\mathrm{NO}_{2}$ successfully suppressed myocardial damage, MPO activity levels and ICAM-1 expression levels. Furthermore, the suppression of inflammation induced by CAPE- $\mathrm{NO}_{2}$ was mediated via the downregulation of MPO and ICAM-1 expression levels. To the best of our knowledge, these results suggest for the first time that CAPE- $\mathrm{NO}_{2}$ has anti-inflammatory activity in IR-induced myocardial injury.

In addition to the antioxidant and anti-inflammatory effects of CAPE- $\mathrm{NO}_{2}$ against IR-induced cardiac injury, increased anti-apoptotic effects were also observed in accordance with activation of the PI3K/Akt/mTOR signaling pathway, which is a potent signaling pathway for survival associated with several diseases, including IR injury (14-16). The present study demonstrated that the administration of CAPE- $\mathrm{NO}_{2}$ significantly increased the expression levels of p-PI3K, p-Akt and p-mTOR in the myocardium. These data suggested that $\mathrm{CAPE}-\mathrm{NO}_{2}$-induced prevention of myocardial IR injury may depend, at least in part, on the PI3K/Akt/mTOR pathway. Akt exerts its protective effects through the phosphorylation of diverse molecular targets, including the Bcl-2 family (17). Previous studies have suggested that IR may stimulate the apoptosis of cardiomyocytes via the downregulation of Bcl-2 with a simultaneous increase in Bax expression levels $(2,4)$. Furthermore, the Bax/Bcl-2 ratio may be a critical factor in the cellular threshold for apoptosis (18). Cleaved caspase-3 and P38 are two key mediators of apoptosis and are often used as apoptotic markers (6). The activity of caspase- 3 is induced by pro-apoptotic Bax and inhibited by anti-apoptotic Bcl-2 (19). The results of the present study demonstrated that IR increased apoptosis by promoting the expression of Bax, cleaved-caspase-3 and P38 and inhibiting the production of $\mathrm{Bcl}-2$ in cardiomyocytes. Furthermore, CAPE and CAPE- $\mathrm{NO}_{2}$ attenuated IR-induced myocardial apoptosis by simultaneously downregulating Bax, cleaved caspase- 3 and P38, and upregulating Bcl-2 in the cardiomyocytes.

In conclusion, the present in vivo study demonstrated the effectiveness of CAPE- $\mathrm{NO}_{2}$ in attenuating IR-induced myocardial injury, possibly through a reduction in oxidative stress, inflammation and apoptotic cell death, which is in agreement with its potential antioxidant activity. The present study suggests that CAPE- $\mathrm{NO}_{2}$ may be an alternative treatment for oxidation-associated heart diseases.

\section{Acknowledgements}

The authors of the present study would like to thank the National Natural Science Foundation of China (grant no. 21002081) and the Key Program Projects of the Municipal Natural Science Foundation of Chongqing, China (grant no. CSTC2008AA1001) for financial support.

\section{References}

1. Parlakpinar H, Sahna E, Acet A, Mizrak B and Polat A: Protective effect of caffeic acid phenethyl ester (CAPE) on myocardial ischemia-reperfusion-induced apoptotic cell death. Toxicology 209: 1-14, 2005.

2. Liu H, Guo X, Chu Y and Lu S: Heart protective effects and mechanism of quercetin preconditioning on anti-myocardial ischemia reperfusion (IR) injuries in rats. Gene 545: 149-155, 2014.

3. Wei B, Li WW, Ji J, Hu QH and Ji H: The cardioprotective effect of sodium tanshinone IIA sulfonate and the optimizing of therapeutic time window in myocardial ischemia/reperfusion injury in rats. Atherosclerosis 235: 318-327, 2014.

4. Yin Y, Guan Y, Duan J, Wei G, Zhu Y, Quan W, Guo C, Zhou D, Wang Y, Xi M and Wen A: Cardioprotective effect of Danshensu against myocardial ischemia/reperfusion injury and inhibits apoptosis of H9c2 cardiomyocytes via Akt and ERK1/2 phosphorylation. Eur J Pharmacol 699: 219-226, 2013.

5. Quan W, Wu B, Bai Y, Zhang X, Yin J, Xi M, Guan Y, Shao Q, Chen Y, Wu Q and Wen A: Magnesium lithospermate B improves myocardial function and prevents simulated ischemia/reperfusion injury-induced $\mathrm{H} 9 \mathrm{c} 2$ cardiomyocytes apoptosis through Akt-dependent pathway. J Ethnopharmacol 151: 714-721, 2014.

6. Forbes-Hernández TY, Giampieri F, Gasparrini M, Mazzoni L, Quiles JL, Alvarez-Suarez JM and Battino M: The effects of bioactive compounds from plant foods on mitochondrial function: A focus on apoptotic mechanisms. Food Chem Toxicol 68: 154-182, 2014.

7. Zhou K, Li X, Du Q, Li D, Hu M, Yang X, Jiang Q and Li Z: A CAPE analogue as novel antiplatelet agent efficiently inhibits collagen-induced platelet aggregation. Pharmazie 69: 615-620, 2014.

8. Liu TL, Li D, Du Q, Li C, Xiaohua L and Li Z: Novel caffeic acid phenethyl ester (CAPE) analogues as immunoregulatory agents: Synthesis and SAR study. Lat Am J Pharm 32: 329-334, 2013.

9. Parlakpinar H, Ozer MK and Acet A: Effects of captopril and angiotensin II receptor blockers (AT1, AT2) on myocardial ischemia-reperfusion induced infarct size. Cytokine 56: 688-694, 2011

10. Galang N, Sasaki H and Maulik N: Apoptotic cell death during ischemia/reperfusion and its attenuation by antioxidant therapy. Toxicology 148: 111-118, 2000.

11. Harrison D, Griendling KK, Landmesser U, Hornig B and Drexler H: Role of oxidative stress in atherosclerosis. Am J Cardiol 91: 7A-11A, 2003.

12. Jiang WL, Zhang SM, Tang XX and Liu HZ: Protective roles of cornuside in acute myocardial ischemia and reperfusion injury in rats. Phytomedicine 18: 266-271, 2011.

13. Campo GM, Avenoso A, Campo S, Nastasi G, Traina P, D'Ascola A, Rugolo CA and Calatroni A: The antioxidant activity of chondroitin-4-sulphate, in carbon tetrachloride-induced acute hepatitis in mice, involves NF-kappaB and caspase activation. Br J Pharmacol 155: 945-956, 2008.

14. Koh PO: Ferulic acid prevents the cerebral ischemic injury-induced decrease of Akt and Bad phosphorylation. Neurosci Lett 507: 156-160, 2012.

15. Zhu M, Feng J, Lucchinetti E, Fischer G, Xu L, Pedrazzini T, Schaub MC and Zaugg M: Ischemic postconditioning protects remodeled myocardium via the $\mathrm{PI} 3 \mathrm{~K}-\mathrm{PKB} / \mathrm{Akt}$ reperfusion injury salvage kinase pathway. Cardiovasc Res 72: 152-162, 2006.

16. Zhan JK, Wang YJ, Wang Y, Wang S, Tan P, Huang W and Liu YS: The mammalian target of rapamycin signalling pathway is involved in osteoblastic differentiation of vascular smooth muscle cells. Can J Cardiol 30: 568-575, 2014.

17. Liu X, Chen Y, Wu Y, Ha T and Li C: The cardioprotection induced by lipopolysaccharide involves phosphoinositide 3-kinase/Akt and high mobility group box 1 pathways. J Biomed Res 24: 324-331, 2010.

18. Chen M, Li B, Zhao X, Zuo H, He X, Li Z, Liu X and Chen L: Effect of diallyl trisulfide derivatives on the induction of apoptosis in human prostate cancer PC-3 cells. Mol Cell Biochem 363: 75-84, 2012.

19. Morkuniene R, Arandarcikaite O and Borutaite V: Estradiol prevents release of cytochrome $c$ from mitochondria and inhibits ischemia-induced apoptosis in perfused heart. Exp Gerontol 41: 704-708, 2006. 